\title{
Pulmonary and intranasal delivery of thymoquinone-loaded nanoparticles for
}

\section{Mucormycosis \& Covid-19}

Andrei P. Sommer, ${ }^{1 *}$ Horst-Dieter Försterling, ${ }^{2}$ Kurt G. Naber, ${ }^{3}$ Ramasamy M. Pitchappan ${ }^{4}$

${ }^{I}$ Faculty of Science, Isra University, Amman, Jordan.

${ }^{2}$ Department of Chemistry, Philipps University Marburg, Marburg, Germany.

${ }^{3}$ Department of Urology, Technical University of Munich, Munich, Germany.

${ }^{4}$ Department of Immunology, Madurai Kamaraj University, Madurai, India.

Submitted: June 3, 2021

Accepted: June 16, 2021

Published: June 25, 2021

\section{Graphical Abstract}

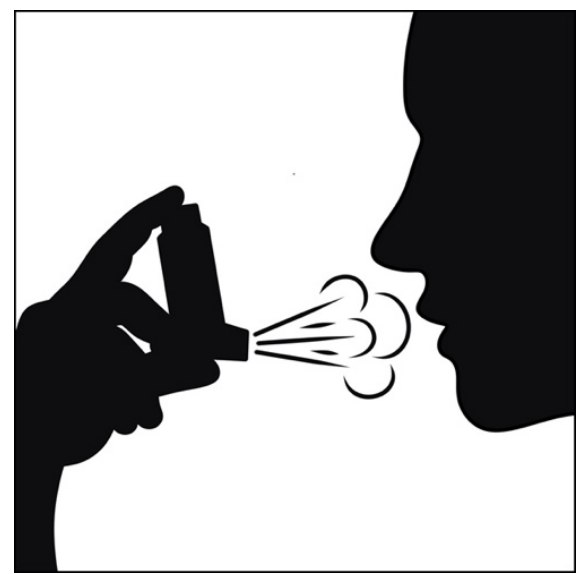

Two potentially life-saving strategies based on pulmonary and intranasal administration of thymoquinone-loaded nanoparticles to treat mucormycosis, an opportunistic infection, which is expanding exponentially in immunocompromised Covid-19 patients in India.

\section{Abstract}

India is currently reporting a massive increase of mucormycosis infection among Covid-19 patients. The opportunistic black fungus infection affects the lungs and the brain. Depending on the infected organs, morbidity and mortality rates are between $50 \%$ and $100 \%$. To save patients' life, it is frequently necessary to remove jaws and eyes. The drug of the first choice for the treatment of mucormycosis in the subcontinent is amphotericin B. There are two problems with amphotericin B: First, it is currently not available in many pharmacies in India, and second, its effect is restricted by its limited passage across the blood-brain barrier. Recently, it was shown that thymol is highly effective against Rhizopus oryzea, the fungus causing $70 \%$ of mucormycosis. In contrast to amphotericin $\mathrm{B}$, which in the case of mucormycosis is administered intravenously, thymol can reach the lungs via inhalation and the brain via the nose-to-brain route. Importantly, thymoquinone (TQ) has all the relevant biological properties of thymol and has already been successfully used to treat Covid-19, as demonstrated in clinical studies. In addition to the action spectrum of thymol, TQ can act as a reactive oxygen species and oxidize pathogens upon contact, thereby recommending itself for both treatment of Covid-19 and infections with the black fungus. For maximum efficacy, comprising biodistribution, bioavailability, and drug functionality, we describe innovative strategies based on nanomedicines for targeted delivery of TQ to the lungs and intranasal delivery to the brain, bypassing the blood-brain barrier. After these promising strategies as the next step, a clinical study for proof of concept should be initiated.

${ }^{*}$ Dr. Andrei P. Sommer, Visiting Professor, Department of Chemistry, Faculty of Science, ISRA University, Amman, Jordan, andrei.sommer@alumni.uni-ulm.de 


\section{Keywords}

Mucormycosis, Covid-19, coronavirus, SARS-CoV-2, Rhizopus oryzea, predictive modeling, nanomedicines, pulmonary delivery, intranasal delivery, thymol, thymoquinone

\section{Purpose and Rationale}

Considering the antiviral, antibacterial, antiinflammatory, and immunomodulatory effects of thymoquinone (TQ), four properties, which are essential for both prophylaxis treatment of Covid-19, we recommended the utilization of the drug against the new coronavirus. Our approach received support from the examination of the physicochemical properties of the SARS-CoV-2 virus and the TQ molecule, leading to a model describing the interaction of TQ with the virus both in the extra- and in intracellular space. This model was further confirmed in silico as well as in clinical trials. Here, we extend the predictive capability of this model by including the impact of TQ on Rhizopus oryzea. Exploiting the action spectrum of TQ and the existing arsenal of nanocarriers, it becomes clear that pulmonary and intranasal administration of TQ to immunocompromised Covid-19 patients infected with mucormycosis could prove to be a successful therapeutic against this lifethreatening, rapidly spreading secondary infection after Covid-19.

\section{Introduction}

Severely ill Covid-19 patients can develop fungal infections during the middle and latter stages of their disease [1]. In a recent article, Post coronavirus disease mucormycosis: a deadly addition to the pandemic spectrum, Sharma et al. suggested that extensive use of steroids in the management of Covid-19 might facilitate the emergence of the infection mucormycosis by suppressing the immunity [2], a mold infection resulting in high morbidity and mortality rates [3]. The primary route and cause of this opportunistic infection is the inhalation of spores of the species Rhizopus oryzea, abundant in the environment. Besides rhino-cerebral and pulmonary invasion, spores distributed via the bloodstream can affect various organs. Mucormycosis also affects the skin (black fungus), the eyes, jaw bone, and the gastrointestinal tract (colonic perforation).

Steroids are used to minimize the damage in severe Covid-19 infections. They reduce inflammation in the lungs and thus limit the damage caused by the cytokine storm. While steroid administration is a life-saving intervention for critically ill Covid-19 patients, it also reduces immunity. Pathological conditions, which are favoring mucormycosis are neutropenia and diabetes mellitus [4]. An intact immune system can easily eliminate incorporated spores of the fungus, but not a suppressed one. According to the literature, fatality in mucormycosis is high: If the fungus is limited to the sinuses, the fatality rate is 40 45 percent, if it invades the lungs, 70-80 percent, and if it spreads to the brain, sinuses, and lungs, 90-100 percent. It is thus imperative that antifungal therapy is started at an early stage of the infection to save the patient with limited damage.

\section{Materials and Methods}

India is currently reporting a rash of this otherwise rare infection among Covid-19 patients. Black fungus cases are reported all over India, and the incidences are expected to increase exponentially in the days to come [5]. The government of India has mobilized amphotericin B, the drug of the first choice for the treatment of mucormycosis in the subcontinent. Since its synthesis in 1955, the drug has been successfully used worldwide to treat such systemic fungal infections. One major problem with this drug involves its pharmacokinetics, including its limited passage across the blood-brain barrier (BBB) [6] - a limitation inherent to most drugs circulating in the blood - and thus making the time and stage of intervention crucial in saving lives. Hence, the need of the hour is a drug with a broad action spectrum, and the potential to (I) kill spores of Rhizopus oryzea circulating in the blood and seeded in organs, (II) enter and clear the sinuses and the lungs, (III) enter the brain bypassing the $\mathrm{BBB}$, and (IV) be used topically. It is known that nose-to-brain drug delivery is feasible through a unique connection present between the olfactory epithelium and the trigeminal nerve, thus bypassing the BBB $[7,8]$. The drug thymoquinone (TQ) can be a good candidate in this direction, offering a viable alternative to amphotericin B.

Using predictive modeling, we recently recommended TQ for both the prophylaxis and treatment of Covid-19 [9,10]. Several theoretical calculations [11-13] and clinical 
trials [14-16] have confirmed the predictive capability of our model. The integral protective properties and relevant physicochemical functions executed by TQ in both the extra- and intracellular space have been recently compiled [17]: In short, TQ is a small hydrophobic molecule with the capacity to bind to the viral spikes, to bind to the viral envelope, to oxidize the virus, to scavenge reactive oxygen species (ROS) - after oxidation of the virus - and to enter cells and raise endosomal $\mathrm{pH}$. In addition to its antiviral potential, demonstrated in vitro using human-derived SARS-CoV-2 [18], TQ presents broad antibacterial, anti-inflammatory, and immunomodulatory effects. A representative list can be found in $[9,10]$ and the literature within.

Furthermore, TQ presents strong fungicidal activity based on ROS [19]. The physicochemical properties of TQ and clinical factors recommending this natural compound for Covid-19 turn out to recommend it for mucormycosis too, thus offering a practical alternative to the actual routine: Interestingly, Rhizopus oryzea strains tested 2012 in Brazil were found to be resistant to amphotericin B, however not to the essential oil of Thymus vulgaris and its constituent thymol, which were shown to significantly inhibit mycelial growth and germination of sporangiospores [20]. Thymol, a chemical precursor of TQ, is used to synthesize the latter on industrial scales. TQ presents virtually all the biologically relevant properties of thymol. Additionally, it can act as a ROS (oxidize) - a key parameter in fighting fungi, bacteria, and viruses, in general, and in combating SARS-CoV-2. This functional analogy and toxicological superiority strongly favor the implementation of TQ in the therapy of mucormycosis.

This is where nanoformulations come into play: TQ is a hydrophobic molecule and has limited bioavailability and biodistribution, particularly in organs such as the lungs and the brain.

For pulmonary drug delivery, we shall consider here only two instructive solutions: The first consists of the use of large (micronsized) porous particles. This approach is inspired by the insight that when particles are too small, an army of alveolar macrophages is phagocytosing them after their landing in the deep lungs. In theory, phagocytic clearance could be avoided by tuning the size of the particles. However, by virtue of their larger size and corresponding weight, for large particles, the probability of losses increases before entering the lower airways. This fundamental contrast in physical parameters explains the increased aerosolization efficiency observed for large, light particles. The merits of this combination for pulmonary drug delivery were assessed by encapsulated model therapeutics inside large porous particles composed of 50:50 poly(lactic acid-co-glycolic acid) [21]. The second solution, plausible, consist of the use of nanocarriers and modes of administration, which maximize the drug concentration at the alveoli of the lungs. This strategy safeguards that pathogen (spores, fungi, and viruses) can be neutralized before they invade the cells. For effective destruction of the pathogens, the delivery of TQ is ideally carried out with capsules facilitating an instant release of the cargo. A comprehensive overview of nanoparticles for pulmonary delivery is provided in [22] and for pulmonary delivery of TQ in [23].

In contrast to the strategies for pulmonary drug delivery, intranasal drug delivery favors nanoparticles [24]. Instructive models exploring nanoformulations for TQ delivery, in general, and to the brain are provided in [25] and [26], respectively. Technically, the administration of nanoparticles (nanosuspensions) can be realized using asthma sprays or inhalers [27].

\section{Results and Discussion}

Amidst the pandemic, in such a short span, a handful of Covid-19 vaccines have been successfully designed, manufactured, and administered to high-risk individuals all over the world: It is an unfinished agenda that will take a few more tens of months. Nonetheless, the discovery of many new virulent mutants in different parts of the world is a matter of concern: whether a vaccine designed for an earlier virus and the resultant immunity will work for new variants is still a question. In contrast to the Covid-19 vaccines, the mechanism of action of the nutraceutical TQ safeguards the non-specific neutralization of the coronavirus and its variants. This difference between the modalities of action provides a heuristic justification for the simultaneous treatment of Covid-19 and mucormycosis with 
TQ, thereby taking advantage of its ROS-like function [28-30]. Choosing this strategy is further supported by the fact that administration of TQ is associated with the consolidation of the immune system $[9,10]$ - perhaps the most critical aspect in the therapy of Covid-19.

Thus, the rapid spread of the black fungus in India brought the drug TQ one more time into the limelight, this time as a potential treatment of both conditions Covid-19 and mucormycosis. The indication for the use of this natural compound in the treatment of this aggressive disease receives immediate justification: The drug can be administered in three different ways: (I) ingestion for systemic distribution, (II) inhalation via nebulizer using an alcoholic extract of Nigella sativa oil targeting both the sinuses and lungs and (III) topical use of the oil. These liquid and vaporbased administration forms, allowing for a considerable therapeutic reach, were recently extended by TQ in solid form. Last fall, Intas, one of the leading multinational pharmaceutical formulation development, manufacturing, and marketing companies in the world, introduced Thymotas - the world's first ready-to-use TQ tablet [31]. The tablets are available in India, marking the beginning of a significant

\section{Conclusion}

In contrast to the narrow and highly specific biological effect of the vaccines, whose function is activation and conditioning immunity, TQ neutralizes the virus non-specifically via a physicochemical mechanism, a crucial aspect, which has not been considered so far by the medical community. The authors express their hope that this short article will initiate a discussion and a proof-of-concept clinical study with a favorable turn in the Indian and World bodies and set an alternative trend to treat mucormycosis.

\section{Acknowledgment}

APS is grateful to Noah Lotan (Technion, Haifa, Israel) and Sripriya Jaganathan for fruitful discussions. The authors thank Katlin E. Sommer for designing the graphical content.

\section{Conflict of Interests}

The authors declare that there are no conflicts of interest. For signed statements, please contact the journal office: editor@precisionnanomedicine.com

\section{Author contributions}

Design of study: APS; Writing: APS with input from all authors. Methodology: APS and HDF; Substantial revision of the manuscript: KGN and RMP. All authors discussed the results and commented on the Manuscript.

Refer to this article as Sommer AP, Försterling HD, Naber KG, Pitchappan RM, Pulmonary and intranasal delivery of thymoquinone-loaded nanoparticles for Mucormycosis and Covid-19, Precis. Nanomed. 2021;4(2):795-800, https://doi.org/10.33218/001c.24963 


\section{References}

1. Gangneux JP, Bougnoux ME, Dannaoui E, Cornet M, Zahar JR. Invasive fungal diseases during COVID-19: we should be prepared. J Mycol Med. 2020;30:100971.

2. Sharma S, Grover M, Bhargava S, Samdani S, Kataria T. Post coronavirus disease mucormycosis: a deadly addition to the pandemic spectrum. J Laryngol Otol. 2021 Apr 8; 1-6, Online ahead of print.

3. Prakash H, Chakrabarti A. Global epidemiology of mucormycosis. J Fungi (Basel). 2019;5:26.

4. Morales-Franco B, Nava-Villalba M, Medina-Guerrero EO, Sánchez-Nuño YA, Davila-Villa P, Anaya-Ambriz EJ, et al. Host-pathogen molecular factors contribute to the pathogenesis of Rhizopus spp. in diabetes mellitus. Curr Trop Med Rep. 2021 Jan 22;1-12.

5. The Tribune, June 8 , https://www.tribuneindia.com/news/nation/28-252-black-fungus-cases-inindia-265262

6. Chikley A, Ronen Ben-Ami R, Kontoyiannis DP. Mucormycosis of the central nervous system. J Fungi (Basel). 2019;5:59.

7. Chowdhury FA, Hossain MK, Mostofa AGM, Akbor MM, Bin Sayeed MS. Therapeutic potential of thymoquinone in glioblastoma treatment: Targeting major gliomagenesis signaling pathways. Biomed Res Int. 2018:4010629.

8. Hanson LR, Frey II WH Intranasal delivery bypasses the blood-brain barrier to target therapeutic agents to the central nervous system and treat neurodegenerative disease. BMC Neuroscience. 2008;9(Suppl 3):S5.

9. Sommer AP, Försterling HD, Naber KG. Thymoquinone: Shield and sword against SARS-CoV-2. Precis Nanomed. 2020;3:541-548.

10. Sommer AP, Försterling HD, Sommer KE. Tutankhamun's antimalarial drug for Covid-19. Drug Res (Stuttg). 2021;71:4-9.

11. Srivastava N, Garg P, Srivastava P, Seth PK. A molecular dynamics simulation study of the ACE2 receptor with screened natural inhibitors to identify novel drug candidate against COVID-19. PeerJ. 2021;9:e11171.

12. Badary OA, Hamza MS, Tikamdas R. Thymoquinone: A promising natural compound with potential benefits for COVID-19 prevention and cure. Drug Des Devel Ther. 2021;15:1819-1833.

13. Xu H, Liu B, Xiao Z, Zhou M, Ge L, Jia F, et al. Computational and experimental studies reveal that thymoquinone blocks the entry of coronaviruses into in vitro cells. Infect Dis Ther. 2021;10:483-494.

14. El Sayed SM, Aboonq MS, El Rashedy AG, Aljehani YT, Abou El-Magd RM, Okashah AM, et al. Promising preventive and therapeutic effects of TaibUVID nutritional supplements for COVID-19 pandemic: towards better public prophylaxis and treatment (A retrospective study). Am J Blood Res. 2020;10:266-282.

15. Ashraf S, Ashraf S, Ashraf M, Imran MA, Kalsoom L, Siddiqui UN, et al. Honey and Nigella sativa against COVID-19 in Pakistan (HNS-COVID-PK): A multi-center placebo-controlled randomized clinical trial. MedRxiv preprint, doi.org/10.1101/2020.10.30.20217364.

16. Koshak AE, Koshak EA, Mobeireek AF, Badawi MA, Wali SO, Malibary HM, et al. Nigella sativa supplementation accelerates recovery from mild COVID-19: First randomized controlled clinical trial (RCT). OSF preprints, DOI:10.31219/osf.io/urb6f.

17. Sommer AP. https://isbe-online.org/files/file/202103/31141003512085.pdf

18. Seadawy MG, Gad AF, Elhoseny MF, ELharty BE, Shamel MD, Elfiky AA, et al. In vitro: Natural compounds (thymol, carvacrol, hesperidine, and thymoquinone) against Sars-Cov2 strain isolated from Egyptian patients. bioRxiv preprint, doi.org/10.1101/2020.11.07.367649.

19. Almshawit H, Macreadie I. Fungicidal effect of thymoquinone involves generation of oxidative stress in Candida glabrata. Microbiol Res. 2017;195:81-88.

20. de Lira Mota KS, de Oliveira Pereira F, de Oliveira WA, Lima IO, de Oliveira Lima E. Antifungal activity of Thymus vulgaris L. essential oil and its constituent phytochemicals against Rhizopus oryzae: interaction with ergosterol. Molecules. 2012;17:14418-14433.

21. Edwards DA, Hanes J, Caponetti G, Hrkach J, Ben-Jebria A, Eskew ML, et al. Large porous particles for pulmonary drug delivery. Science. 1997;276:1868-1871. 
22. Amararathna M, Goralski K, Hoskin DW, Rupasinghe HPV. Pulmonary Nano-DrugDelivery Systems for Lung Cancer: Current Knowledge and Prospects. J Lung HealthDis. 2019;3:11-28.

23. Schneider-Stock R, Fakhoury IH, Zaki AM, El-Baba CO, Gali-Muhtasib HU. Thymoquinone: fifty years of success in the battle against cancer models. Drug Discovery Today. 2014;19:18-30.

24. Costa CP, Moreira JN, Sousa Lobo JM, Silva AC. Intranasal delivery of nanostructured lipid carriers, solid lipid nanoparticles and nanoemulsions: A current overview of in vivo studies. Acta Pharm Sin B. 2021;11:925-940.

25. Ballout F, Habli Z, Rahal ON, Fatfat M, Gali-Muhtasib H. Thymoquinone-based nanotechnology for cancer therapy: promises and challenges. Drug Discov Today. 2018;23:1089-1098.

26. Alam S, Khan ZI, Mustafa G, Kumar M, Islam F, Bhatnagar A, Ahmad FJ. Development and evaluation of thymoquinone-encapsulated chitosan nanoparticles for nose-to-brain targeting: a pharmacoscintigraphic study. Int J Nanomedicine. 2012;7:5705-5718.

27. Sommer AP, Försterling HD, Naber KG. Thymoquinone for the treatment or prevention of asymptomatic viral infections. European Patent Application EP20195859.2 2020 Sep 11.

28. Dahlgren C, Karlsson A, Bylund J. Intracellular neutrophil oxidants: From laboratory curiosity to clinical reality. J Immunol. 2019;202:3127-3134.

29. Aguirre J, Hansberg W, Navarro R. Fungal responses to reactive oxygen species. Med Mycol. 2006;44(Suppl 1):S101-S107.

30. Warris A, Ballou ER. Oxidative responses and fungal infection biology. Semin Cell Dev Biol. 2019;89:34-46.

31. https://www.thymotas.com

32. Ribes JA, Vanover-Sams CL, Baker DJ. Zygomycetes in human disease. Clin Microbiol Rev. 2000;13:236-301.

33. Greenberg RN, Scott LJ, Vaughn HH, Ribes JA. Zygomycosis (mucormycosis): Emerging clinical importance and new treatments. Curr Opin Infect Dis. 2004;17:517-525.

34. Schmall E. In the Wake of India's Covid Crisis, a 'Black Fungus' Epidemic Follows. https://www.nytimes.com/2021/06/20/world/asia/india-covid-black-fungus.html

35. Xiao XY, Zhu YX, Bu JY, Li GW, Zhou JH, Zhou SP. Evaluation of Neuroprotective Effect of Thymoquinone Nanoformulation in the Rodent Cerebral Ischemia-Reperfusion Model. Biomed Res Int. 2016;2016:2571060.

36. Kassab RB, El-Hennamy RE.The role of thymoquinone as a potent antioxidant in ameliorating the neurotoxic effect of sodium arsenate in female rat Egypt J Basic Appl Sci. 2017;4:160-167.

37. Abdo W, Elmadawy MA, Abdelhiee EY, Abdel-Kareem MA, Farag A, Aboubakr M et al. Protective effect of thymoquinone against lung intoxication induced by malathion inhalation. Sci Rep. 2021;1:2498. 